\title{
Endoscopic airway equipment part 2: equipment overview
}

\author{
R Hofmeyr, (iD) A Elhouni iD
}

Department of Anaesthesia and Perioperative Medicine, Faculty of Health Sciences, University of Cape Town; and Groote Schuur Hospital, Cape Town, South Africa

Corresponding author, email: ross.hofmeyr@uct.ac.za

\section{Summary}

Endoscopic devices designed for intubation include indirect optical and video laryngoscopes, rigid fibreoptic and video stylets, flexible and rigid bronchoscopes, and optic-enabled intubating supraglottic airways. Various types of surgical operating telescopes can also be used in specific circumstances. It is crucial to understand the strengths and weaknesses of different devices and blade types in order to match the device to the clinical challenge.

Keywords: airway management, anaesthesia equipment, endoscopy, intubation, bronchoscopy

This review is intended to be read in conjunction with the accompanying "Endoscopic airway equipment part 1: physical principles." An earlier unreferenced version was published as course notes accompanying the 2017 FCA 1 Refresher course workshop of the same title.

\section{Introduction}

Safe, reliable airway management is integral to the practice of anaesthesia. ${ }^{1}$ While some procedures may be accomplished without advanced instrumentation of the airway, there are many in which the ability to accurately position endotracheal and endobronchial tubes, bronchial blockers, dilators and other devices is crucial to success, and ultimately, patient outcomes. ${ }^{2}$ The steady growth and refinement of endoscopes used to see within the airway has developed from simple tubes and reflected light-sources to sophisticated hybrid devices using fibre-optics and integrated video camera systems. In addition to a basic understanding of the physics that underlies the function of these devices, it is essential to understand the types, purposes and ideal applications of the wide variety of equipment available. This review aims to give a broad overview of the main types of endoscopic airway equipment in use. More information, training materials and video tutorials can be found on the open-access airway education web site, www.openairway.org. Specific queries can be directed to the authors.

\section{Types of equipment for airway endoscopy}

Devices used for airway endoscopy can be classified by the site of desired use (eg. laryngoscopes, bronchoscopes), the method used to transmit an image (eg. direct, standard optics, fibreoptic or video), and specific properties of the design (eg. rigid or flexible). No single system of classification exists for all the available devices. Furthermore, in specific circumstances, equipment from disciplines outside of anaesthesia is used in complex airway procedures. For instance, rigid telescopes or suspension laryngoscopes are often used in particularly challenging upper airway conditions. In this review, various types of direct laryngoscope are not discussed, as this information is broadly available elsewhere.

Endoscopes for intubation can be divided into indirect (optical or video) laryngoscopes, and endoscopes designed to be placed through an endotracheal tube. Examples of the latter class are flexible fibreoptic bronchoscopes (or flexible intubating video endoscopes), and rigid intubating endoscopes, such as the Bonfils or Shikani Optical Stylet.3-8 The video laryngoscopic devices (and similar optical laryngoscopes) are described in

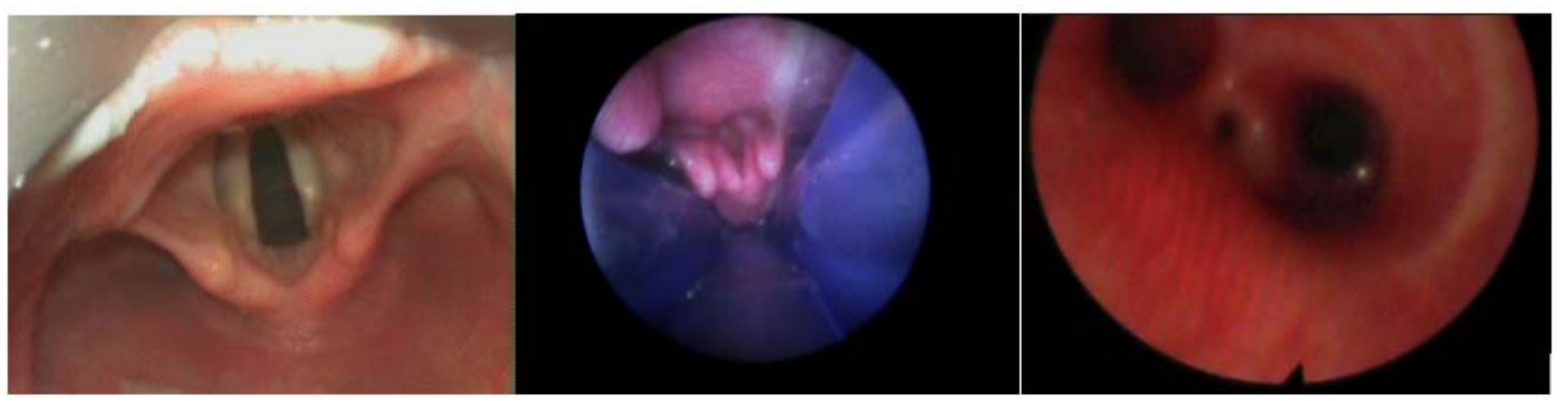

Figure 1: Endoscopic views of the airway. Left to right: video laryngoscope, rigid endoscope through supraglottic airway, flexible video endoscope 
(a)

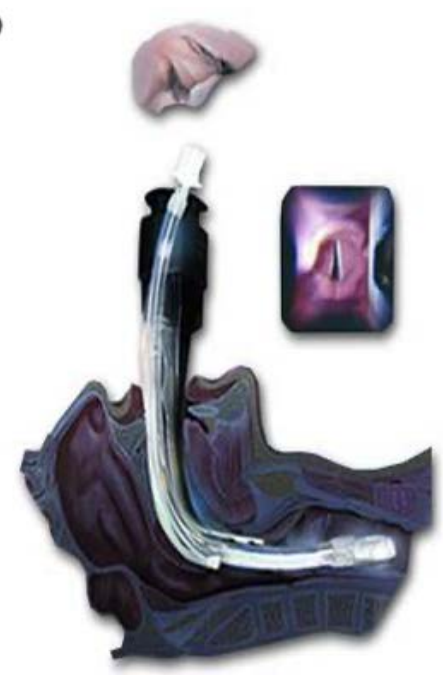

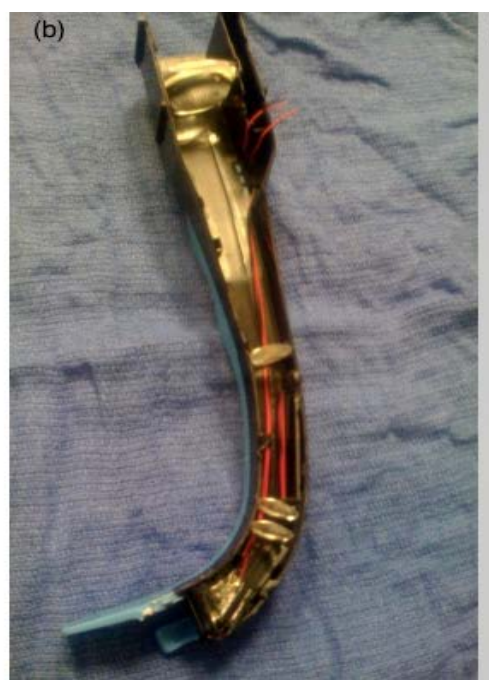

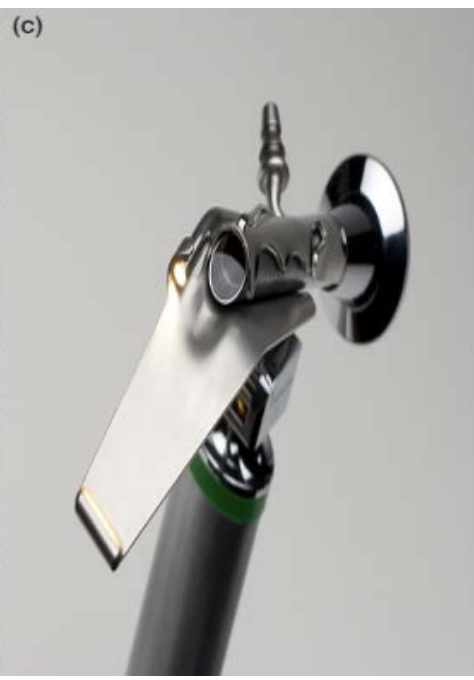

Figure 2: (Above) (a) and (b) Airtraq ${ }^{\circledR}$ optical laryngoscope, showing use and internal construction of lenses, with electrical wires for the LED light source. Note the channelled blade shape. (c) Truview ${ }^{\otimes}$ optical laryngoscope. Images: Manufacturers

the separate section on video laryngoscopy. There are also devices which incorporate video endoscopy into intubating laryngeal masks, such as the $\mathrm{CTrach}^{\mathrm{TM}}$ and the TotalTrack VLM.9-17

\section{Laryngoscopes}

\section{Optical laryngoscopes}

Optical laryngoscopes use lenses, prisms and mirrors to convey the image to an eyepiece, creating an indirect view of the larynx, which allows guided intubation. They often have the advantage of being either low cost and disposable, or compact and robust. All the current devices on the market can have some form of camera connected to the eyepiece to allow display on a screen, forming a hybrid optical/video device. Examples of this type of device include the Airtraq ${ }^{\oplus 18}$ and TruView ${ }^{\otimes}$ optical laryngoscopes. ${ }^{18-21}$

\section{Video laryngoscopes}

The number of video laryngoscope (VL) devices on the market has been rapidly increasing. All use some form of complementary metal-oxide semiconductor (CMOS) or charge-coupled device (CCD) camera chip and lightemitting diode (LED) light source and display the image either on a separate screen or display mounted on the handle. Both disposable and reusable blade devices exist. VLs can be classified according to their blade shape into three groups, which dictates their strengths, weaknesses and utility in different airway situations:

- Hyperangulated blades, such as the classic Glidescope or CMAC Dörges ("D") blade, which are ideally suited to anatomically difficult airways;

Table I: (Below) Properties of endoscopic airway equipment
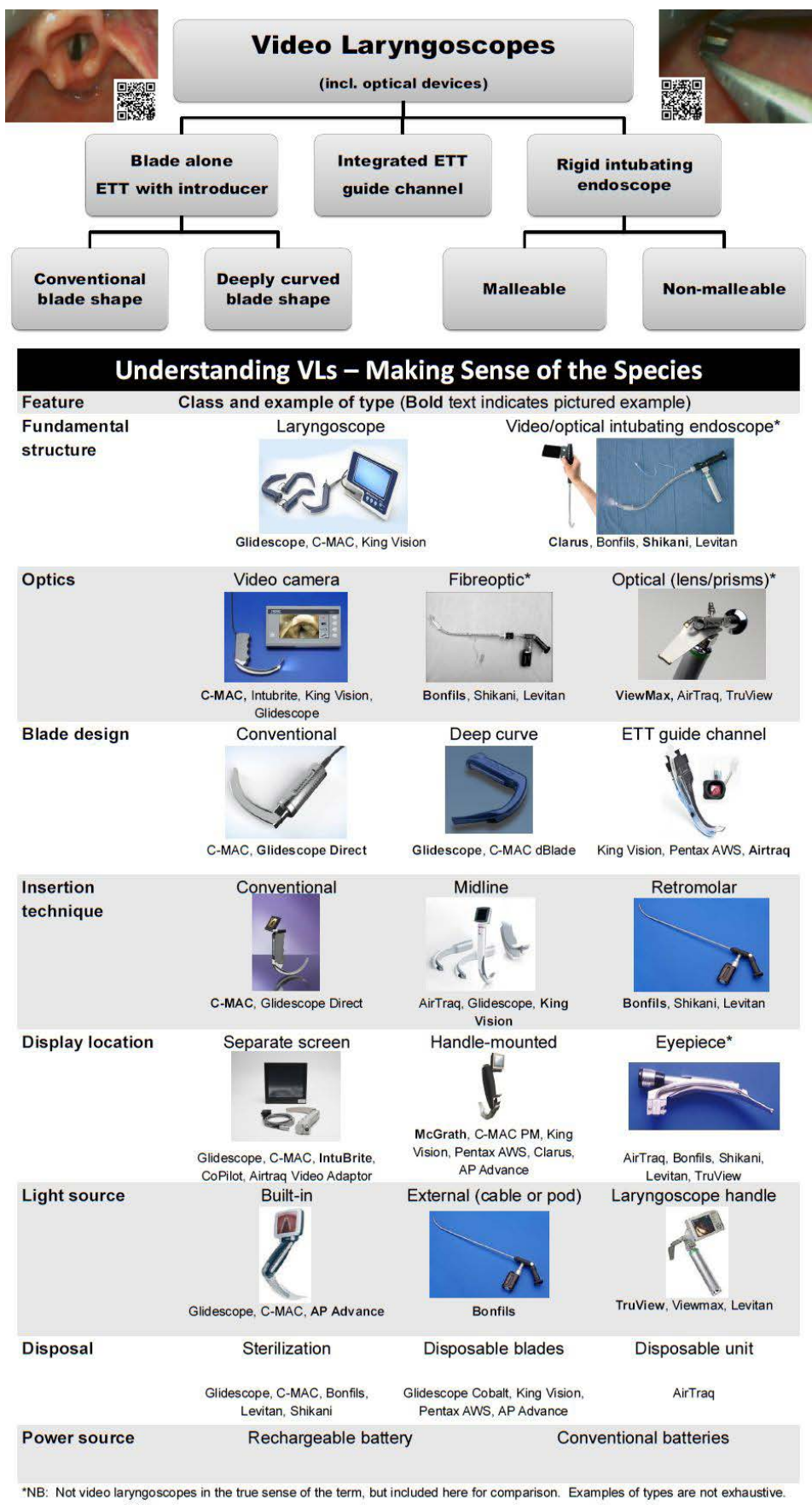


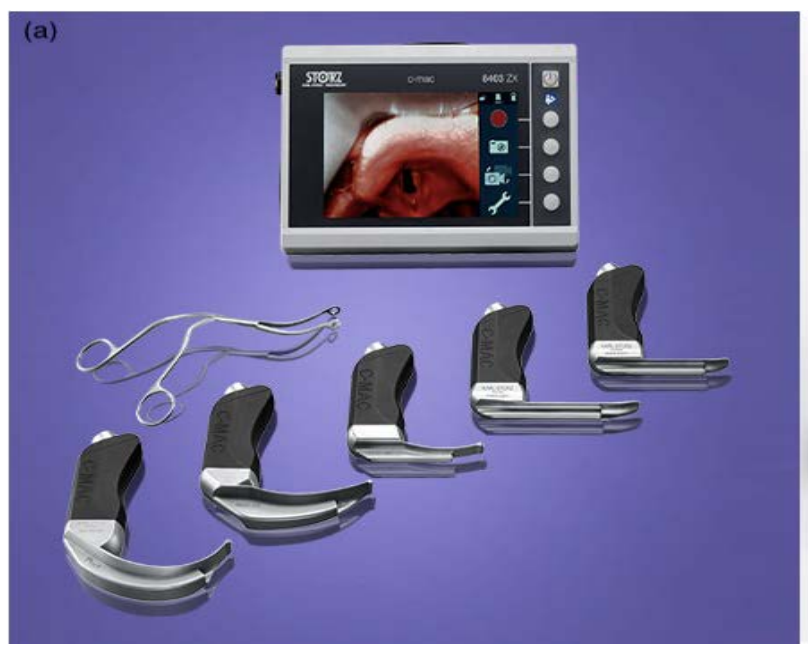

(b)

Figure 3: (a) A set of video laryngoscopes, showing hyperangulated and conventional Macintosh and Miller blade shapes with a separate video display. (b) King Vision ${ }^{\circledR}$ VL with channelled blades and display mounted on handle. Images: Manufacturer

- Traditional Macintosh or Miller shaped blades, which provide improved vision for routine intubation and are ideal for emergency procedures and enhanced training;

- Channelled blades, such as the Pentax AWS or King Vision ${ }^{\oplus}$ VL, which function very well in conditions with airway swelling and are best in providing intubation with minimal head and neck movement.

\section{Rigid intubating endoscopes}

Also known as optical or video stylets, these devices are preloaded with an endotracheal tube and provide a 'through the tube' view during intubation. They are designed to be used alone, or in conjunction with a laryngoscope (known as dual endoscopy). Common examples include the Bonfils, Shikani and Levitan optical stylets, and the Clarus and CMAC-VS video stylets. ${ }^{3,6-8,22}$ The optical stylets use fibreoptic bundles for illumination and image creation, but are much more robust than flexible fibreoptic scopes due to their rigid construction. Due to this, they are manufactured in sizes down to an external diameter of $2 \mathrm{~mm}$, allowing paediatric tubes of as small as 2.5 $\mathrm{mm}$ ID to be used. These devices have the further advantage (in very soiled airways) of being able to be used as a lightwand if intubation under vision is not possible.

Rigid intubating endoscopes are designed to be loaded with the tip of the scope just behind the tip of the tube. This allows a view through the device during intubation. Two techniques can be used: midline, and retromolar. For the midline technique, one hand lifts jaw, or a normal direct laryngoscopy is used to lift the tongue, which aids difficult intubation where there is limited view of the vocal cords or even epiglottis. In patients with limited

mouth opening, or obstructions of the floor of the mouth (such as tumours or submandibular abscess), a retromolar technique can be employed, whereby the scope is passed posteriorly to the molars from the side of the mouth, and is brought into the midline from an oblique angle. Rigid intubating endoscopes are not designed to be passed beyond the vocal cords, but rather to obtain a view of the glottic opening and then allow the tube to be slid into the trachea.

\section{Flexible endoscopes}

Flexible 'scopes can be either designed for bronchoscopy, or as dedicated flexible intubating endoscopes. They are divided into the fibreoptic endoscopes, such as the well-known flexible fibreoptic bronchoscope (FOB), and video endoscopes, where an

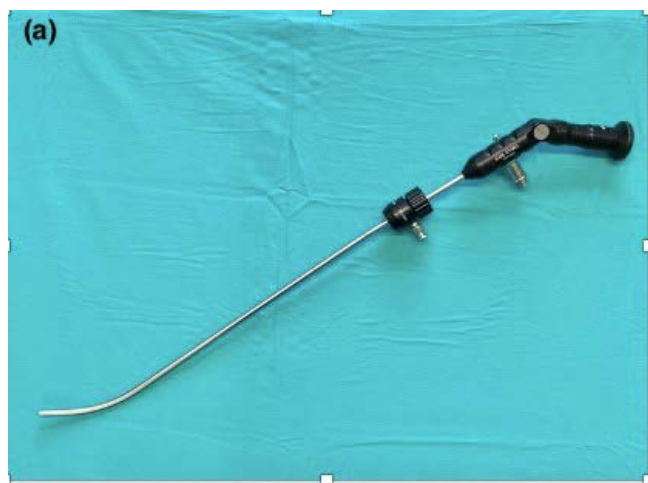

(c)

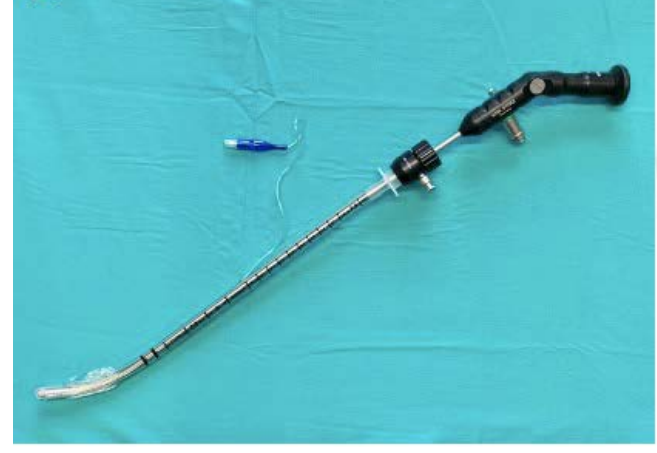

Figure 4: (a) Bonfils (b) Clarus rigid intubating endoscopes (c) Bonfils rigid intubating endoscope with preloaded endotracheal tube and batter light source (b)

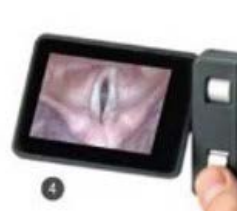

○

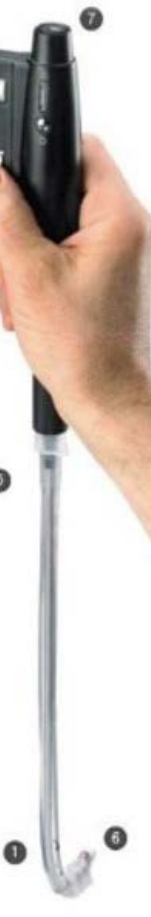




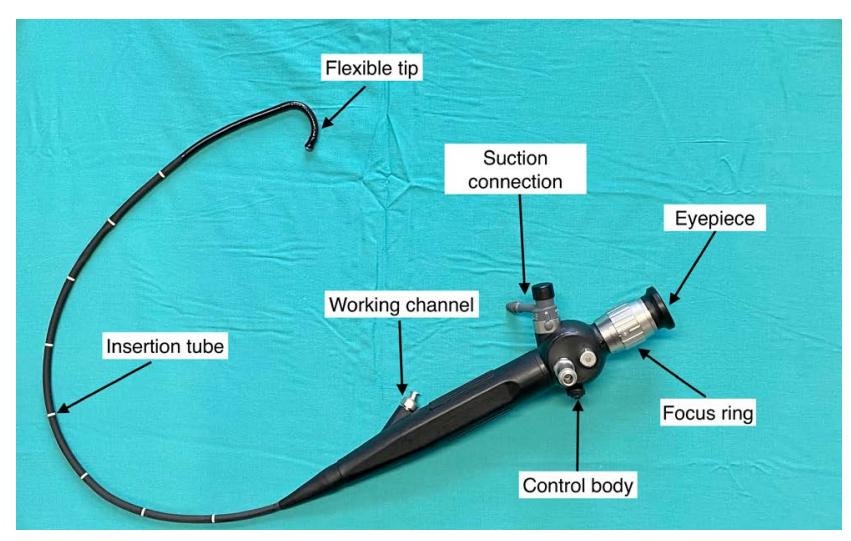

Figure 5: Features of a modern flexible fibreoptic intubating endoscope or bronchoscope

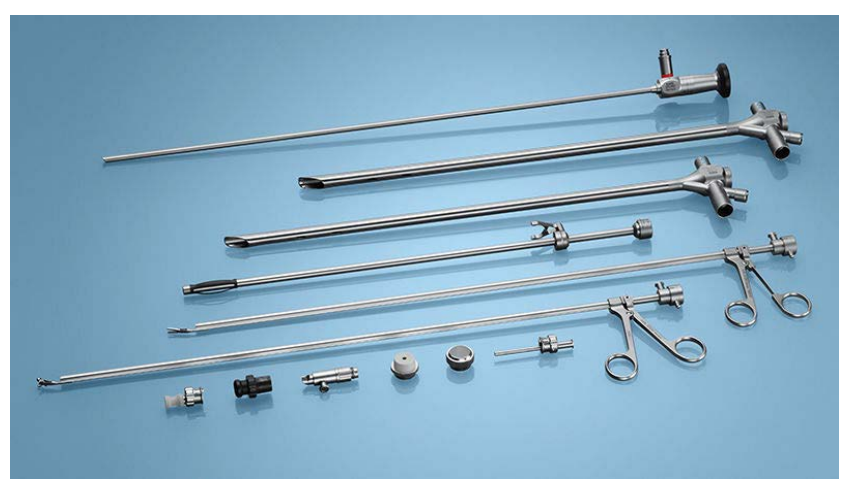

Figure 6: Comprehensive system for rigid bronchoscopy, including rigid telescope, several bronchoscopes, graspers, and attachments for jet and conventional ventilation as well as light delivery

LED light source and "chip-in-tip" video camera are used to give light and create an image. Because video intubating endoscopes do not contain the delicate fibreoptic bundles, they tend to have a larger working channel and be more robust. However, these devices are still delicate pieces of equipment which require very careful care and cleaning.

Flexible intubating endoscopes are designed for awake intubation of very challenging airways. ${ }^{2}$ Functionally, they bear very close resemblance to flexible bronchoscopes (see Figure 5), and are often used interchangeably. Modern models have an interface between the control body and insertion tube (or an additional accessory) to securely hold a preloaded endotracheal tube during endoscopy for intubation. Typically, the minimum internal diameter of the endotracheal tube (ETT) should be no less than $1 \mathrm{~mm}$ greater than the diameter of the scope.

\section{Bronchoscopes}

\section{Rigid bronchoscopes}

Essentially a straight metal tube with connections to allow passage of light, rigid telescopes and operating instruments, rigid bronchoscopes can be used with the naked eye (with a fibreoptic light source connected directly to the scope with a prism) or with a telescopic camera. They are useful to gain access to the airway when swelling or external compression causes collapse or obstruction, and offer a large diameter

(a) working area for surgical tasks and removal of foreign bodies. Rigid tracheoscopes are a slightly shorter variant without side holes, which allows ventilation through the scope while working.

\section{Flexible bronchoscopes}

Practically speaking, there are trivial differences between flexible intubating endoscopes and flexible bronchoscopes. The former are usually slightly $(\sim 5 \mathrm{~cm})$ shorter and slimmer $(5.0-5.5 \mathrm{~mm}$ external diameter), and the latter place larger emphasis on having a larger working channel for graspers and biopsy forceps at the expense of a slight increase in diameter $(5.8-6.3 \mathrm{~mm})$. 'Paediatric' versions of both scopes exist, with usual external diameters of 3.0-4.0 mm. However, considering the implications of scope diameter for endotracheal tube size selection, they function perfectly well for intubation.

\section{Operative telescopes}

Originally, endoscopic telescopes had a traditional lens design, which featured small lenses separated by large air gaps. Physicist Harold Hopkins realised, however, that an alternate design in which the lenses where long rods separated by small airspaces was more efficient, and did not require structures to hold the lenses in place, increasing their size and optical efficiency. This increased the image quality, brightness, and field of vision, while at the same time making the endoscope more robust. After patenting his design in 1959, he was approached by the German optical instrument maker, Herr Karl Storz, to incorporate the design into his endoscopes. This was a turning point for both men; Hopkins became famous for the design, and Storz grew his company into one of the foremost manufacturers of rigid endoscopes in the world. Most modern operative telescopes now use this design, which can be used to create scopes with diameters as small as $1.2 \mathrm{~mm}$ while still producing images far better than those achieved with today's chip-in-tip sensors.
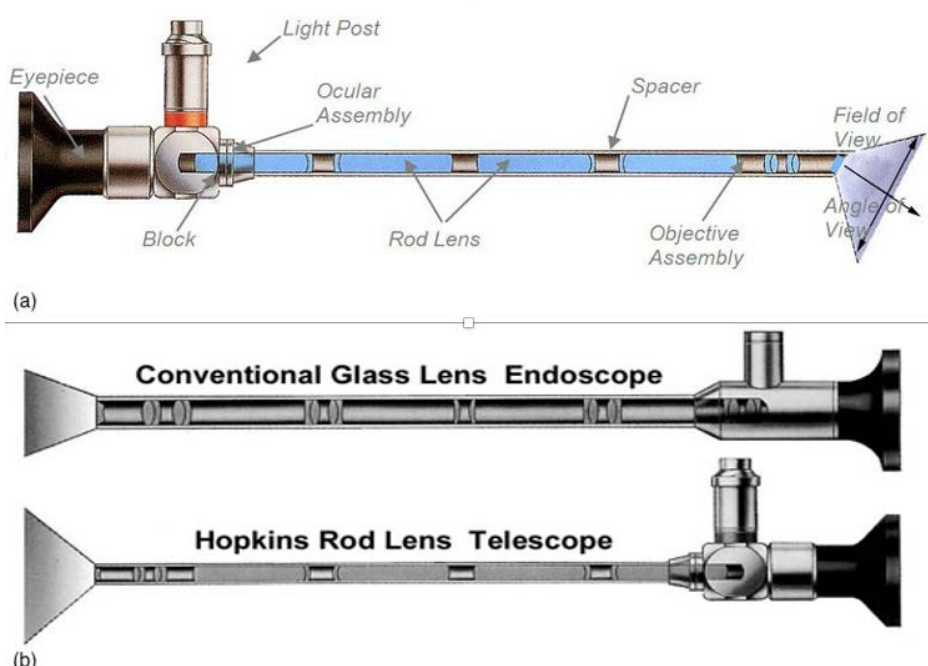

Figure 7: (a) Typical construction of an operative endoscopic telescope, utilising a rod lens design. Image courtesy of Fuzhou Alpha Optics (b) Conventional operative telescope (endoscope) in comparison with the Hopkins 'rod lens' design. Image courtesy of Karl Storz GmBH 
The newest systems for endoscopic surgery incorporate threedimensional (3D) views, providing depth-of-field information to surgeons and reducing the fatigue associated with operating in three dimensions on a two-dimensional screen. Approaches include the use of dual cameras and stereoscopic or polarised screens. Older models use dedicated glasses to provide the 3D effect, but the latest displays allow 3D view without augmentation. Whether this technology is required for anaesthetic purposes remains to be seen.

\section{Care and cleaning}

Cleaning and disinfection of endoscopes is a frequent cause of damage. In this setting, the rigid intubating scopes perform much better, as they are far more robust than any type of flexible 'scope. Flexible'scopes need to be flushed carefully by suctioning a large quantity of warm water or saline through their working channel, wiped clean, and then be placed in an enzymatic detergent to break down any protein build-up or blood clots that may have formed. Thereafter, they are rinsed again and sterilised briefly in a sterilising solution such as Medis ${ }^{\circledR}$ or Cidex ${ }^{\circledast}$. Most hospitals have an infectious disease control protocol which describes the cleaning process for their 'scopes, and information on this process is also available with recommendations from the various manufacturers.

\section{The future}

Clearly, endoscopic airway devices are continuing to evolve, and with them, our surgical and anaesthetic techniques. Patients previously deemed very difficult airways for whom only an awake fibreoptic intubation was advocated are now routinely intubated with asleep video laryngoscopy, and procedures such as tracheal dilatation, which were only performed with rigid bronchoscopes and bougies, are now being achieved as flexible endoscopic day cases through a supraglottic airway. Just as many procedures in cardiac surgery are not achievable without an 'echo anaesthetist' who can provide intraoperative echocardiographic views, so too are procedures in ENT and thoracic surgery beginning to require an 'interventional anaesthetist' who can both control and image the airway. However, quantifying the contribution of these novel and advanced techniques to a wide base of patient outcomes remains a challenge. It is incumbent upon individual practitioners to become highly skilled with the tools at their disposal, and to recognise the relative strengths and weaknesses of each device in each situation.

\section{Conflict of interest}

$\mathrm{RH}$ directs the UCT-Storz African Fellowship in Airway and Thoracic Anaesthesia, which is supported in part by an unrestricted educational grant to the University of Cape Town by Karl Storz GmBH. AE is the incumbent Fellow at the time of writing. No commercial entity had any role in the production or publication of this manuscript. Inclusion of images or mention of specific products by name in this article is for the purposes of discussion and is not an endorsement of the product.

\section{ORCID}

\section{R Hofmeyr (iD https://orcid.org/0000-0002-9990-7459}

\section{A Elhouni (iD https://orcid.org/0000-0003-4970-6592}

\section{References}

1. Frerk C, Mitchell VS, McNarry AF, et al. Difficult Airway Society 2015 guidelines for management of unanticipated difficult intubation in adults. Br J Anaesth. 2015;115(6):827-48. https://doi.org/10.1093/bja/aev371.

2. Ahmad I, El-Boghdadly K, Bhagrath R, et al. Difficult Airway Society guidelines for awake tracheal intubation (ATI) in adults. Anaesthesia. 2020;75(4):509-28. https:// doi.org/10.1111/anae.14904.

3. Halligan $M$, Charters P. A clinical evaluation of the Bonfils intubation fibrescope. Anaesthesia. 2003;58(11):1087-91. https://doi. org/10.1046/j.1365-2044.2003.03407.x.

4. Wong $P$, Lawrence $C$, Pearce $A$. Intubation times for using the Bonfils intubation fibrescope. Br J Anaesth. 2003;91(5):757-8. https://doi.org/10.1093/bja/aeg641.

5. Liao X, Xue FS, Zhang YM, Byhahn C, Meininger D. Tracheal intubation using the Bonfils intubation fibrescope in patients with a difficult airway. Can J Anaesth 2008;55(9):655-6; author reply 6-7. https://doi.org/10.1007/BF03021442.

6. Corbanese $U$, Morossi M. The Bonfils intubation fibrescope: clinical evaluation and consideration of the learning curve. Eur J Anaesthesiol. 2009;26(7):622-4. https://doi.org/10.1097/EJA.0b013e328328f572.

7. Shukry M, Hanson RD, Koveleskie JR, Ramadhyani U. Management of the difficult pediatric airway with Shikani optical stylet. Paediatr Anaesth. 2005;15(4):342-5. https://doi.org/10.1111/j.1460-9592.2005.01435.x.

8. Young CF, Vadivelu N. Does the use of a laryngoscope facilitate orotracheal intubation with a Shikani optical stylet? Br J Anaesth. 2007;99(2):302-3. https:// doi.org/10.1093/bja/aem196

9. Bjerkelund CE. Use of a new intubating laryngeal mask-CTrach-in patients with known difficult airways. Acta Anaesthesiol Scand. 2006;50(3):388. https://doi. org/10.1111/j.1399-6576.2006.00915.x

10. Goldman AJ, Wender R, Rosenblatt W, Theil D. The fiberoptic intubating LMA-CTrach: an initial device evaluation. Anesth Analg. 2006;103(2):508. https:// doi.org/10.1213/01.ANE.0000227214.33583.23.

11. Liu EH, Goy RW. The LMA CTrach for unanticipated difficult intubation. Anaesthesia. 2006;61(10):1015. https://doi. org/10.1111/j.1365-2044.2006.04810.x.

12. Liu EH, Goy RW, Chen FG. The LMA CTrach, a new laryngeal mask airway for endotracheal intubation under vision: evaluation in 100 patients. $\mathrm{Br} J$ Anaesth. 2006;96(3):396-400. https://doi.org/10.1093/bja/ael001.

13. Timmermann A, Russo S, Graf BM. Evaluation of the CTrach-an intubating LMA with integrated fibreoptic system. Br J Anaesth. 2006;96(4):516-21. https://doi. org/10.1093/bja/ael029.

14. Chilkoti GT, Agarwal M, Mohta M, et al. A randomised preliminary study to compare the performance of fibreoptic bronchoscope and laryngeal mask airway CTrach (LMA CTrach) for visualisation of laryngeal structures at the end of thyroidectomy. Indian J Anaesth. 2020;64(8):704-9. https://doi.org/10.4103/ ija.IJA_138_20.

15. Gomez-Rios MA, Freire-Vila E, Vizcaino-Martinez L, Estevez-Gonzalez E. The Totaltrack: an initial evaluation. Br J Anaesth. 2015;115(5):799-800. https://doi. org/10.1093/bja/aev336.

16. Choonoo J, Hofmeyr R, Evans N, James M, Meyersfeld N. A new option in airway management: an evaluation of the TotalTrack ${ }^{\circledast}$ videolaryngeal mask. Br J Anaesth. 2016;117:114-5.

17. Gomez-Rios MA, Bonome C. The TotalTrack VLM: a novel video-assisted intubating laryngeal mask. Minerva Anestesiol. 2018;84(1):126-7.

18. Maharaj CH, O'Croinin D, Curley G, Harte BH, Laffey JG. A comparison of tracheal intubation using the Airtraq or the Macintosh laryngoscope in routine airway management: A randomised, controlled clinical trial. Anaesthesia. 2006;61(11):1093-9. https://doi.org/10.1111/j.1365-2044.2006.04819.x.

19. Ford PNR, Hamer C, Medakkar S. Use of the Airtraq in the difficult airway. Eur J Anaesthesiol. 2007;24(8):730-1. https://doi.org/10.1017/S0265021507000233.

20. Hoshijima $H$, Mihara T, Denawa $Y$, et al. Airtraq ${ }^{\oplus}$ is superior to the Macintosh laryngoscope for tracheal intubation: systematic review with trial sequential analysis. Am J Emerg Med. 2019;37(7):1367-8. https://doi.org/10.1016/j. ajem.2018.12.018.

21. Nasim S, Maharaj CH, Butt I, et al. Comparison of the Airtraq ${ }^{\circledR}$ and Truview laryngoscopes to the Macintosh laryngoscope for use by Advanced Paramedics in easy and simulated difficult intubation in manikins. BMC Emerg Med. 2009;9:2. https://doi.org/10.1186/1471-227X-9-2.

22. 22. Bein $B$, Yan $M$, Tonner $P H$, Steinfath $M$, Dörges V. Tracheal intubation using the Bonfils intubation fibrescope after failed direct laryngoscopy. Anaesthesia. 2004;59(12):1207-9. https://doi.org/10.1111/j.1365-2044.2004.03967.x. 


\section{רeleflex}

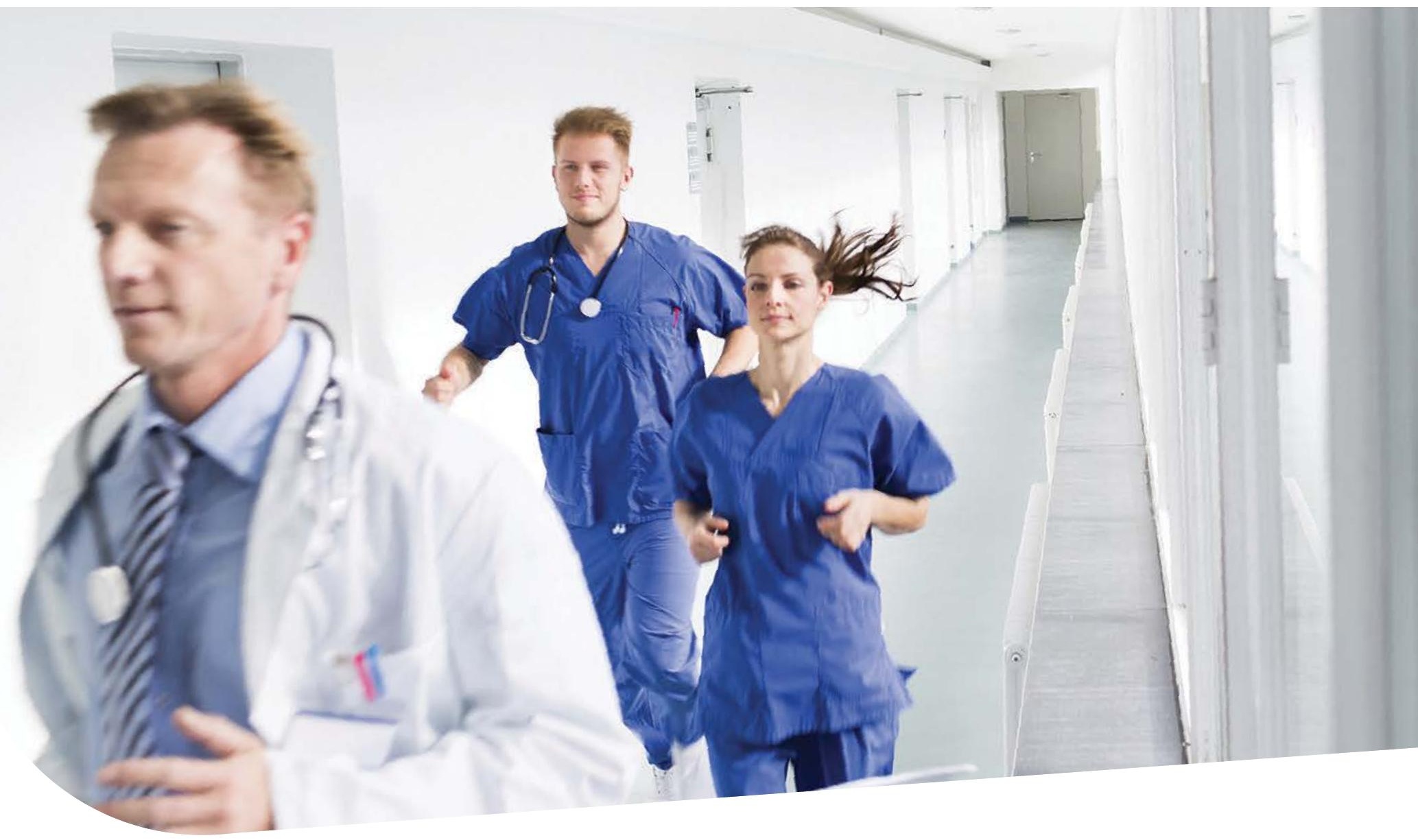

\section{Uniting best in class technologies that deliver critical care solutions.}

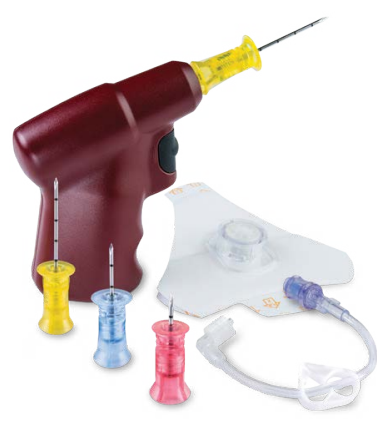

Arrow EZ-IO Intraosseous Vascular Access System

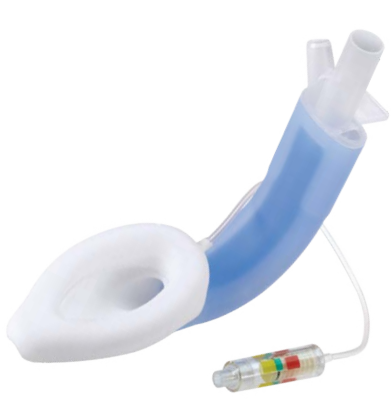

LMA Protector Airway Laryngeal Mask

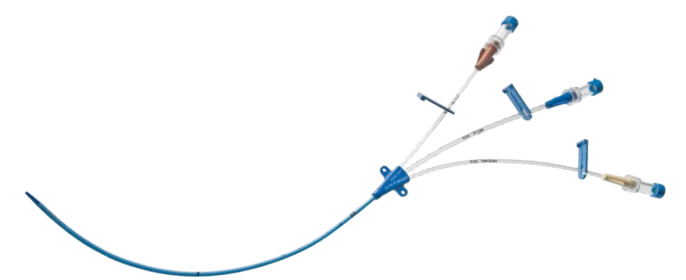

ARROWg ${ }^{+}$ard Blue Central Venous Catheter 